\title{
THE HILALI CONJECTURE ON PRODUCT OF SPACES
}

\author{
SHOJI YOKURA
}

\begin{abstract}
The Hilali conjecture claims that a simply connected rationally elliptic space $X$ satisfies the inequality $\operatorname{dim}\left(\pi_{*}(X) \otimes \mathbb{Q}\right) \leqq \operatorname{dim} H_{*}(X ; \mathbb{Q})$. In this paper we show that for any such space $X$ there exists a positive integer $n_{0}$ such that for any $n \geqq n_{0}$ the strict inequality $\operatorname{dim}\left(\pi_{*}\left(X^{n}\right) \otimes \mathbb{Q}\right)<\operatorname{dim} H_{*}\left(X^{n} ; \mathbb{Q}\right)$ holds, where $X^{n}$ is the product of $n$ copies of $X$.
\end{abstract}

\section{INTRODUCTION}

The most important and fundamental topological invariant in geometry and topology is the Euler-Poincaré characteristic $\chi(X)$, which is defined to be the alternating sum of the Betti numbers $\beta_{i}(X):=\operatorname{dim} H_{i}(X ; \mathbb{Q})$ :

$$
\chi(X):=\sum_{i \geqq 0}(-1)^{i} \beta_{i}(X),
$$

provided that each $\beta_{i}(X)$ and $\chi(X)$ are both finite. Similarly, for a topological space whose fundamental group is an Abelian group one can define the homotopical Betti number $\beta_{i}^{\pi}(X):=$ $\operatorname{dim}\left(\pi_{i}(X) \otimes \mathbb{Q}\right)$ where $i \geqq 1$ and the homotopical Euler-Poincaré characteristic:

$$
\chi^{\pi}(X):=\sum_{i \geqq 1}(-1)^{i} \beta_{i}^{\pi}(X),
$$

provided that each $\beta_{i}^{\pi}(X)$ and $\chi^{\pi}(X)$ are both finite. The Euler-Poincaré characteristic is the special value of the Poincaré polynomial $P_{X}(t)$ at $t=-1$ and the homotopical EulerPoincaré characteristic is the special value of the homotopical Poincaré polynomial $P_{X}^{\pi}(t)$ at $t=-1$ :

$$
\begin{aligned}
P_{X}(t) & :=\sum_{i \geqq 0} t^{i} \beta_{i}(X), \quad \chi(X)=P_{X}(-1), \\
P_{X}^{\pi}(t) & :=\sum_{i \geqq 1} t^{i} \beta_{i}^{\pi}(X), \quad \chi^{\pi}(X)=P_{X}^{\pi}(-1) .
\end{aligned}
$$

From now on any topological space is assumed to be path-connected, unless otherwise stated. The well-known Hilali conjecture 2 2 claims that for a simply connected rationally elliptic space $X$ (i.e.., both $\operatorname{dim}\left(\pi_{*}(X) \otimes \mathbb{Q}\right)<\infty$ and $\operatorname{dim} H_{*}(X ; \mathbb{Q})<\infty$ ), then

$$
\operatorname{dim}\left(\pi_{*}(X) \otimes \mathbb{Q}\right) \leqq \operatorname{dim} H_{*}(X ; \mathbb{Q}), \quad \text { namely, } \quad P_{X}^{\pi}(1) \leqq P_{X}(1) .
$$

Here $\pi_{*}(X) \otimes \mathbb{Q}:=\sum_{i \geqq 1} \pi_{i}(X) \otimes \mathbb{Q}$ and $H_{*}(X ; \mathbb{Q}):=\sum_{i \geqq 0} H_{i}(X ; \mathbb{Q})$. No counterexample to the Hilali conjecture has been so far found yet.

In this paper we show that for a simply connected rationally elliptic space $X$ there exists a positive integer $n_{0}$ such that for $\forall n \geqq n_{0}$

$$
\operatorname{dim}\left(\pi_{*}\left(X^{n}\right) \otimes \mathbb{Q}\right)<\operatorname{dim} H_{*}\left(X^{n} ; \mathbb{Q}\right) .
$$

Here $X^{n}$ is the product $X^{n}=\underbrace{X \times \cdots \times X}_{n}$.

2010 MSC: 55P62, 55Q40, 55N99.

Keywords: Hilali conjecture, rational homotopy theory. 
Remark 1.1. In the Hilali conjecture a topological space $X$ is required to be simply connected, i.e., the fundamental group is trivial, $\pi_{1}(X)=0$. One of the reason of this requirement is that in general the fundamental group is not an Abelian group, thus one cannot define tensoring $\pi_{1}(X) \otimes \mathbb{Q}$. In fact, you do need the condition of simply connectedness. The well-known counterexample is $S^{1} \vee S^{2}$. Since $H_{0}\left(S^{1} \vee S^{2}\right)=H_{1}\left(S^{1} \vee S^{2}\right)=H_{1}\left(S^{1} \vee S^{2}\right)=\mathbb{Z}$, hence $\operatorname{dim} H_{*}\left(S^{1} \vee S^{2} ; \mathbb{Q}\right)=3$. However $\pi_{1}\left(S^{1} \vee S^{2}\right)=\mathbb{Z}$ (thus $S^{1} \vee S^{2}$ is not simply connected) and $\pi_{2}\left(S^{1} \vee S^{2}\right)=\mathbb{Z}^{\infty}$. So $\operatorname{dim} \pi_{*}(X) \otimes \mathbb{Q}=\infty$. Hence $S^{1} \vee S^{2}$ is not rationally elliptic. So, a naive question is if the Hilali conjecture still holds for a rationally elliptic space whose fundamental group is an Abelian group.

\section{Poincaré polynomial and homotopical Poincaré polynomial}

The Poincaré polynomial $P_{X}(t)$ is multiplicative in the following sense:

$$
P_{X \times Y}(t)=P_{X}(t) \times P_{Y}(t)
$$

which follows from the Künneth Formula:

$$
H_{n}(X \times Y ; \mathbb{Q})=\sum_{i+j=n} H_{i}(X ; \mathbb{Q}) \otimes H_{j}(Y ; \mathbb{Q}) .
$$

The homotopical Poincaré polynomial $P_{X}^{\pi}(t)$ is additive in the following sense:

$$
P_{X \times Y}^{\pi}(t)=P_{X}^{\pi}(t)+P_{Y}^{\pi}(t)
$$

which follows from

$$
\pi_{i}(X \times Y)=\pi_{i}(X) \times \pi_{i}(Y)=\pi_{i}(X) \oplus \pi_{i}(X) \quad \text { and } \quad(A \oplus B) \otimes \mathbb{Q}=(A \otimes \mathbb{Q}) \oplus(B \otimes \mathbb{Q}) .
$$

Here, for a later use, we compute the Poincaré polynomial and homotopical Poincaré polynomial of spheres. The following are well-known results (due to Serre Finiteness Theorem [3, 4]):

$$
\pi_{i}\left(S^{2 k}\right) \otimes \mathbb{Q}=\left\{\begin{array}{ll}
\mathbb{Q} & i=2 k \\
\mathbb{Q} & i=4 k-1 \\
0 & i \neq 2 k, 4 k-1
\end{array} \quad \pi_{i}\left(S^{2 k+1}\right) \otimes \mathbb{Q}= \begin{cases}\mathbb{Q} & i=2 k+1 \\
0 & i \neq 2 k+1\end{cases}\right.
$$

Hence we have that

$$
P_{S^{2 k+1}}^{\pi}(t)=t^{2 k+1} \text { and } P_{S^{2 k+1}}(t)=t^{2 k+1}+1
$$

Thus we have

$$
P_{S^{2 k+1}}^{\pi}(t)<P_{S^{2 k+1}}(t) \text { for } \forall t
$$

However we have

$$
P_{S^{2 k}}^{\pi}(t)=t^{4 k-1}+t^{2 k} \text { and } P_{S^{2 k}}(t)=t^{2 k}+1
$$

Hence we have

$$
\left\{\begin{array}{cc}
P_{S^{2 k}}^{\pi}(t)<P_{S^{2 k}}(t), & t<1 \\
P_{S^{2 k}}^{\pi}(t)=P_{S^{2 k}}(t), & t=1 \\
P_{S^{2 k}}^{\pi}(t)>P_{S^{2 k}}(t), & t>1 \\
2 &
\end{array}\right.
$$




\section{The Hilali conjecture on products of spheres}

It is known that the Hilali conjecture holds for products of spheres. In this section, first we show the following more general statement, using the multiplicativity of the Poincaré polynomial and the additivity of the homotopical Poincaré polynomial observed above.

First we observe that for a pathconnected space $X \operatorname{dim} H_{*}(X ; \mathbb{Q})=1$ if and only if $X$ is rationally homotopy equivalent to a point, which is due to the fundamental fact that $X \sim_{\mathbb{Q}} Y$ if and only if the Sullivan's minimal models $M_{X}$ and $M_{Y}$ are isomorphic. Another simpler argument is using the well-known Whitehead-Serre Theorem [1, Theorem 8.6]. Indeed, $\operatorname{dim} H_{*}(X ; \mathbb{Q})=1$ for a pathconnected space $\mathrm{X}$ is equivalent to $\left(a_{X}\right)_{*}: H_{*}(X ; \mathbb{Q}) \rightarrow H_{*}(p t)=$ $\mathbb{Q}$ being an isomorphism, where $a_{X}: X \rightarrow p t$ is the map to a point. Thus it follows from the Whitehead-Serre Theorem that $\left(a_{X}\right)_{*}: \pi_{*}(X) \otimes \mathbb{Q} \rightarrow \pi_{*}(p t) \otimes \mathbb{Q}=0$ is an isomorphism. Therefore we get the following strict inequality

$$
0=\operatorname{dim}\left(\pi_{*}(X) \otimes \mathbb{Q}\right)<\operatorname{dim} H_{*}(X ; \mathbb{Q})=1, \quad \text { namely } \quad 0=P_{X}^{\pi}(1)<P_{X}(1)=1
$$

Proposition 3.2. Let $X_{i}(1 \leqq i \leqq n)$ be a rationally elliptic space such that the fundamental group is an Abelian group, then $X_{1} \times \cdots \times X_{n}$ is also rationally elliptic, and if $P_{X_{i}}^{\pi}(1) \leqq P_{X_{i}}(1)$, then $P_{X_{1} \times \cdots \times X_{n}}^{\pi}(1) \leqq P_{X_{1} \times \cdots \times X_{n}}(1)$.

The above observation (3.1) implies that if $X_{i}(1 \leqq i \leqq k)$ satisfies $P_{X_{i}}(1)=\operatorname{dim} H_{*}\left(X_{i} ; \mathbb{Q}\right) \geqq$ 2 and $X_{j}(k+1 \leqq j \leqq n)$ satisfies $P_{X_{j}}(1)=\operatorname{dim} H_{*}\left(X_{j} ; \mathbb{Q}\right)=1$, thus $P_{X_{j}}^{\pi}(1)=0$, then we have

$$
\begin{aligned}
& P_{X_{1} \times \cdots \times X_{n}}^{\pi}(1)=P_{X_{1} \times \cdots \times X_{k}}^{\pi}(1)+P_{X_{k+1}}^{\pi}(1)+\cdots+P_{X_{n}}^{\pi}(1)=P_{X_{1} \times \cdots \times X_{k}}^{\pi}(1) . \\
& P_{X_{1} \times \cdots \times X_{n}}(1)=P_{X_{1} \times \cdots \times X_{k}}(1) \times P_{X_{k+1}}(1) \times \cdots \times P_{X_{n}}(1)=P_{X_{1} \times \cdots \times X_{k}}(1) .
\end{aligned}
$$

(Namely, taking the product by a space $Z$ satisfying $\operatorname{dim} H_{*}(Z ; \mathbb{Q})=1$ does not change the value of $P_{\bullet}^{\pi}(1)$ and $P_{\bullet}(1)$.) Therefore, to prove the proposition, we can assume that for each $X_{i}$ we have $P_{X_{i}}(1) \geqq 2$. Since $P_{X_{1} \times \cdots \times X_{n}}^{\pi}(1)=P_{X_{1}}^{\pi}(1)+\cdots+P_{X_{n}}^{\pi}(1)$ and $P_{X_{1} \times \cdots \times X_{n}}(1)=$ $P_{X_{1}}(1) \cdots P_{X_{n}}(1)$, this proposition follows from the following elementary lemma.

Lemma 3.3. Let $a_{i}, b_{i}(1 \leqq i \leqq n)$ be real numbers such that $a_{i} \leqq b_{i}$ and $2 \leqq b_{i}$ for each $i$. Then we have

$$
a_{1}+a_{2} \cdots+a_{n} \leqq b_{1} b_{2} \cdots b_{n}
$$

In particular, we have

$$
b_{1}+b_{2}+\cdots+b_{n} \leqq b_{1} b_{2} \cdots b_{n} .
$$

Proof. A proof is easy, but for the sake of completeness we write a proof. Let $n=2$. Since $a_{1} \leqq b_{1}$ and $a_{2} \leqq b_{2}$, we have $a_{1}+a_{2} \leqq b_{1}+b_{2}$.

$$
\begin{aligned}
b_{1} b_{2}-\left(b_{1}+b_{2}\right) & =\left(b_{1} b_{2}-b_{1}-b_{2}+1\right)-1 \\
& =\left(b_{1}-1\right)\left(b_{2}-1\right)-1 \\
& \left.\geqq 1 \cdot 1-1=0 \quad \text { (because } b_{i} \geqq 2, \text { thus } b_{i}-1 \geqq 1\right)
\end{aligned}
$$

Hence $b_{1}+b_{2} \leqq b_{1} b_{2}$. Therefore we have $a_{1}+a_{2} \leqq b_{1} b_{2}$. Now, suppose that we have $a_{1}+$ $a_{2}+\cdots+a_{n-1} \leqq b_{1} b_{2} \cdots b_{n-1}$. Since $2 \leqq b_{1} b_{2} \cdots b_{n-1}$, by applying $a_{1}+a_{2} \leqq b_{1} b_{2}$ to the inequalities $a_{1}+a_{2}+\cdots+a_{n-1} \leqq b_{1} b_{2} \cdots b_{n-1}$ and $a_{n} \leqq b_{n}$ we get

$$
\left(a_{1}+a_{2}+\cdots+a_{n-1}\right)+a_{n} \leqq\left(b_{1} b_{2} \cdots b_{n-1}\right) b_{n},
$$

namely we get $a_{1}+a_{2} \cdots+a_{n} \leqq b_{1} b_{2} \cdots b_{n}$. 
As an application of the above proposition we can see that the Hilali conjecture holds for the product of a finite family of spheres of dimension $\geqq 2$. (Note that we need that the dimension of each sphere is $\geqq 2$ because in the Hilali conjecture a space has to be simply connected.) It follows from (2.1) and (2.2) above that for any sphere $S^{n}$ of any dimension $n$ we have

$$
P_{S^{n}}^{\pi}(1) \leqq P_{S^{n}}(1) \quad \text { and } \quad P_{S^{n}}(1)=2
$$

Therefore the following corollary follows from Proposition 3.2 ,

Corollary 3.4. The Hilali conjecture holds for the product $S^{n_{1}} \times S^{n_{2}} \times \cdots \times S^{n_{r}}$ of any finite family of spheres $S^{n_{i}}$ of any dimension $\geqq 2$ :

$$
P_{S^{n_{1}} \times S^{n_{2}} \times \cdots \times S^{n_{r}}}^{\pi}(1) \leqq P_{S^{n_{1}} \times S^{n_{2}} \times \cdots \times S^{n_{r}}}(1) .
$$

Hence the Hilali conjecture also holds for any topological space $S$ which is homotopy equivalent to such a product of spheres:

$$
P_{S}^{\pi}(1) \leqq P_{S}(1)
$$

Remark 3.5. It is clear that the above inequalities hold even if we do not require the dimension of each sphere to be $\geqq 2$.

We can get the following corollary from the above Proposition 3.2 and the above remark:

Corollary 3.6. Let $X$ be a rationally elliptic space such that its fundamental group is an Abelian group. Then $X^{n}:=X \times X \times \cdots \times X$ is also rationally elliptic, and if $P_{X}^{\pi}(1) \leqq P_{X}(1)$, then $P_{X^{n}}^{\pi}(1) \leqq P_{X^{n}}(1)$.

\section{Hilali conjecture "Modulo product"}

Motivated by the above Corollary [3.6, from the multiplicativity of the Poincaré polynomial $P_{X}(1)$ and the additivity of homotopical Poincaré polynomial $P_{X}^{\pi}(1)$ we can get the following theorem from an elementary calculus fact:

Theorem 4.1 (Hilali conjecture "modulo product"). Let $X$ be a rationally elliptic space such that its fundamental group is an Abelian group. Then there exists some integer $n_{0}$ such that for $\forall n \geqq n_{0}$ the following strict inequality holds:

$$
\operatorname{dim}\left(\pi_{*}\left(X^{n}\right) \otimes \mathbb{Q}\right)<\operatorname{dim} H_{*}\left(X^{n} ; \mathbb{Q}\right) \text {, i.e., } P_{X^{n}}^{\pi}(1)<P_{X^{n}}(1) .
$$

Proof. Since $X$ is rationally elliptic, for any integer $X^{n}$ is also rationally elliptic.

If $P_{X}(1)=1$, then it follows from (3.1) that $P_{X}^{\pi}(1)=0$, hence for any integer $n \geqq 1$ we have

$$
0=P_{X^{n}}^{\pi}(1)<P_{X^{n}}(1)=1 .
$$

So, suppose that $P_{X}(1) \geqq 2$. The multiplicativity of the Poincaré polynomial $P_{X}(1)$ and the additivity of homotopical Poincaré polynomial $P_{X}^{\pi}(1)$ imply the following

$$
\frac{P_{X}^{\pi}(1)}{P_{X^{n}}(1)}=\frac{n P_{X}^{\pi}(1)}{\left(P_{X}(1)\right)^{n}}
$$

Since $P_{X}(1) \geqq 2, \frac{1}{P_{X}(1)}<1$. Thus it follows from the elementary calculus 1 that

$$
\lim _{n \rightarrow \infty} n\left(\frac{1}{P_{X}(1)}\right)^{n}=0
$$

\footnotetext{
${ }^{1}$ If $|r|<1$, we have $\lim _{n \rightarrow \infty} n r^{n}=0$.
} 
Therefore, whatever the value $P_{X}^{\pi}(1)$ is, we obtain

$$
\lim _{n \rightarrow \infty} n P_{X}^{\pi}(1)\left(\frac{1}{P_{X}(1)}\right)^{n}=\lim _{n \rightarrow \infty} \frac{n P_{X}^{\pi}(1)}{\left(P_{X}(1)\right)^{n}}=0 .
$$

Hence there exists some integer $n_{0}$ such that for all $n \geqq n_{0}$

$$
\frac{P_{X^{n}}^{\pi}(1)}{P_{X^{n}}(1)}=\frac{n P_{X}^{\pi}(1)}{\left(P_{X}(1)\right)^{n}}<1 .
$$

Therefore there exists some integer $n_{0}$ such that for all $n \geqq n_{0}$

$$
P_{X^{n}}^{\pi}(1)<P_{X^{n}}(1)
$$

Definition 4.2. Let $X$ be a rationally elliptic space such that its fundamental group is an Abelian group.

(1) The minimum integer $n_{0}$ such that

$$
P_{X^{n_{0}}}^{\pi}(1)<P_{X^{n_{0}}}(1)
$$

shall be called the "homology-rank-dominating" power of $X$ and denoted by $\mathfrak{p}_{0}(X)$.

(2) The minimum integer $n_{0}$ such that

$$
P_{X^{n_{0}}}^{\pi}(1) \leqq P_{X^{n_{0}}}(1)
$$

shall be called the "homology-rank-almost-dominating" power of $X$ and denoted by $\mathfrak{p}(X)$.

Remark 4.3. Since $P_{X^{n_{0}}}^{\pi}(1)$ and $P_{X^{n_{0}}}(1)$ are both homotopy invariant, the powers $\mathfrak{p}_{0}(X)$ and $\mathfrak{p}(X)$ are both homotopy invariants

Remark 4.4. The Hilali conjecture claims that the "homology-rank-almost-dominating" power $\mathfrak{p}(X)$ is always 1 .

Example 4.5. Let $n$ be any positive integer. Since $P_{S^{2 n}}^{\pi}(1)=2$ and $P_{S^{2 n}}(1)=2$, we have

$$
\begin{gathered}
P_{S^{2 n} \times S^{2 n}}^{\pi}(1)=2+2=4, P_{S^{2 n} \times S^{2 n}}(1)=2 \times 2=4, \\
P_{S^{2 n} \times S^{2 n} \times S^{2 n}}^{\pi}(1)=2+2+2=6, P_{S^{2 n} \times S^{2 n} \times S^{2 n}}(1)=2 \times 2 \times 2=8 .
\end{gathered}
$$

Hence we have

$$
\mathfrak{p}_{0}\left(S^{2 n}\right)=3 \quad \text { and } \quad \mathfrak{p}\left(S^{2 n}\right)=1 .
$$

Example 4.6. Let $n, m$ be any positive integers. Since $P_{S^{2 n} \times S^{2 m}}^{\pi}(1)=2+2=4$ and $P_{S^{2 n} \times S^{2 m}}(1)=2+2=4$, we have

$$
P_{\left(S^{2 n} \times S^{2 m}\right) \times\left(S^{2 n} \times S^{2 m}\right)}^{\pi}(1)=4+4=8, P_{\left(S^{2 n} \times S^{2 m}\right) \times\left(S^{2 n} \times S^{2 m}\right)}(1)=4 \times 4=16 .
$$

Hence we have

$$
\mathfrak{p}_{0}\left(S^{2 n} \times S^{2 m}\right)=2 \quad \text { and } \quad \mathfrak{p}\left(S^{2 n} \times S^{2 m}\right)=1 .
$$

Remark 4.7. If the Hilali conjecture is correct, then we have the following:

(1) if $\operatorname{dim}\left(\pi_{*}(X) \otimes \mathbb{Q}\right)<\operatorname{dim} H_{*}(X ; \mathbb{Q})$, then $\mathfrak{p}_{0}(X)=1$.

(2) if $\operatorname{dim}\left(\pi_{*}(X) \otimes \mathbb{Q}\right)=\operatorname{dim} H_{*}(X ; \mathbb{Q})=2$, then $\mathfrak{p}_{0}(X)=3$.

(3) if $\operatorname{dim} H_{*}(X ; \mathbb{Q}) \geqq 3$ and $\operatorname{dim}\left(\pi_{*}(X) \otimes \mathbb{Q}\right)=\operatorname{dim} H_{*}(X ; \mathbb{Q})$, then $\mathfrak{p}_{0}(X)=2$. 


\section{A FinAL REMARK}

If we plug in $t=-1$ in the two equations (2.1) and (2.2) in $\S 2$, we get the following

$$
\begin{gathered}
P_{S^{2 k+1}}^{\pi}(-1)=-1<P_{S^{2 k+1}}(-1)=0, \\
P_{S^{2 k}}^{\pi}(-1)=0<P_{S^{2 k}}(-1)=2 .
\end{gathered}
$$

Therefore, for the product $S^{n_{1}} \times S^{n_{2}} \times \cdots \times S^{n_{r}}$ of any finite family of spheres $S^{n_{i}}$ we have

$$
P_{S^{n_{1}} \times S^{n_{2}} \times \cdots \times S^{n_{r}}}^{\pi}(-1) \leqq 0 \quad \text { and } \quad 0 \leqq P_{S^{n_{1}} \times S^{n_{2}} \times \cdots \times S^{n_{r}}}(-1) .
$$

Thus we get the following :

Corollary 5.4. For the product $S^{n_{1}} \times S^{n_{2}} \times \cdots \times S^{n_{r}}$ of any finite family of spheres $S^{n_{i}}$ we have

$$
P_{S^{n_{1}} \times S^{n_{2}} \times \cdots \times S^{n_{r}}}^{\pi}(-1) \leqq P_{S^{n_{1}} \times S^{n_{2}} \times \cdots \times S^{n_{r}}}(-1) .
$$

Hence for any topological space $S$ which is homotopy equivalent to such a product of spheres, we have

$$
P_{S}^{\pi}(-1) \leqq P_{S}(-1)
$$

By the definition it is clear that $P_{X}^{\pi}(0)<P_{X}(0)$. Since -1 is symmetric to 1 as to 0 , the above Corollary 5.4 is in fact a special case of the following proposition, which is a "mirror version" of the Hilali conjecture:

Proposition 5.5. ([1, Proposition 32.10]) Let $X$ be a simply connected rationally elliptic space. Then

$$
\chi^{\pi}(X) \leqq 0 \quad \text { and } \quad 0 \leqq \chi(X)
$$

Namely we have $P_{X}^{\pi}(-1) \leqq P_{X}(-1)$ i.e.,

$$
\sum_{i \geqq 1}(-1)^{i} \operatorname{dim}\left(\pi_{i}(X) \otimes \mathbb{Q}\right) \leqq \sum_{i \geqq 0}(-1)^{i} \operatorname{dim} H_{i}(X ; \mathbb{Q}) .
$$

Remark 5.6. It should be noted that in [1, Proposition 32.10] a bit stronger statement is also given; $\chi^{\pi}(X)=0 \Longleftrightarrow \chi(X)>0$, in other words $\chi^{\pi}(X)<0 \Longleftrightarrow \chi(X)=0$. Indeed, for example, let us consider the above product of finitely many spheres $X:=S^{n_{1}} \times S^{n_{2}} \times \cdots \times S^{n_{r}}$, in which case $\chi^{\pi}(X)=0 \Longleftrightarrow \chi(X)>0$ follows from both (5.1) and (5.2).

Remark 5.7. Finally we remark that in [5] we discuss the ratio $h(X):=\frac{P_{X}^{\pi}(1)}{P_{X}(1)}$ of a fibration $X$ of elliptic spaces. Note that if the Hilali conjecture holds, then $0 \leqq h(X) \leqq 1$.

Acknowledgements: The author would like to thank Toshihiro Yamaguchi for useful comments. This work is supported by JSPS KAKENHI Grant Numbers JP16H03936 and JP19K03468.

\section{REFERENCES}

[1] Y. Félix, S. Halperin and J.-C. Thomas, Rational Homotopy Theory, Graduate Texts in Math., 205, Springer, 2001.

[2] M. R. Hilali, Action du tore Tn sur les espaces simplement connexes, PhD thesis, Universite catholique de Louvain, 1980.

[3] J.-P. Serre, "Homologie singulière des espaces fibrés: Applications", Ann. of Math., 54 (1951), 425-505.

[4] J.-P. Serre, Groupes d'homotopie et classes de groupes abelien, Ann. of Math., 58 (1953), 258-294.

[5] T. Yamaguchi and S. Yokura, On ratios of homotopy and homology ranks of fibrations, preprint, May 2019. 
Department of Mathematics and Computer Science, Graduate School of Science and Engineering, Kagoshima University, 1-21-35 Korimoto, Kagoshima, 890-0065, Japan

E-mail address: yokura@sci.kagoshima-u.ac.jp 